\title{
Incidence and Indices of Keratoconus in Patients presenting for LASIK in Egypt
}

\author{
${ }^{1}$ Mohammed Othman Abdel Khalek El Sayed, ${ }^{2}$ Naglaa Hassan Ali
}

\section{ABSTRACT}

Background: Keratoconus $(\mathrm{KC})$ is a progressive, non-inflammatory bilateral but (usually asymmetrical) ecstatic corneal disease, characterized by paraxial stromal thinning and weakening that leads to corneal surface distortion. The reported incidence and prevalence of $\mathrm{KC}$ varies widely depending upon the geographic location, diagnostic criteria used and the cohort of patients selected.

Aim: To estimate the incidence and indices of $\mathrm{KC}$ in patients seeking refractive procedures in Beni Sueif governorate at a span period of one year from March 2013 to March 2014. To describe and analyze the characteristics of the KC population under study.

Materials and methods: This study as designed as a prospective surveillance study. All clinical records in the computerized database from pentacam with the clinical diagnosis of $\mathrm{KC}$ and / or suspected were included and statistically analyzed.

Results: Data from 2116 cases with the average $28.8 \pm$ 6.5 years old were analyzed over the span of 1 year starting from March 2013 to March 2014. Based on topographic images of the pentacam 36 eyes were diagnosed as $\mathrm{KC}$. The incidence of $\mathrm{KC}$ was $1.7 \%$ ( $95 \%$ confidence interval, 0.55-1.45). In keratoconic patients, the mean age was 28.1 years, the mean $\mathrm{K} 1$ and $\mathrm{K} 2$ were $45.18 \pm 3.44 \mathrm{D}$ and $49.49 \pm 4.28 \mathrm{D}$ respectively. The mean minimal pachymetry value was $463 \pm 6.51$ um, the mean index of surface variance (ISV) was $61 \pm 32$, the mean index of vertical asymmetry (IVA) was $0.63 \pm 0.38$, the mean keratoconus indices $(\mathrm{KI})$ was $1.08 \pm 0.14$.

Conclusion: This study provides the first population-based estimate of the incidence of keratoconus in Beni suef governorate in the upper Egypt. According to this study incidence rate of $\mathrm{KC}$ is comparable to the rate among the Caucasian people in middle Asia e.g. Iran while incidence rate is much higher among Malaysia and Kingdom of Saudi Arabia citizens.

Keywords: Incidence, Indices, Keratoconus.

How to cite this article: El Sayed MOAK, Ali NH. Incidence and Indices of Keratoconus in Patients presenting for LASIK in Egypt. Int J Kerat Ect Cor Dis 2017;6(1):17-22.

\footnotetext{
1,2 Lecturer

${ }^{1}$ Department of Ophthalmology, Faculty of Medicine, Beni Suef University, Beni Suef, Egypt

${ }^{2}$ Department of Ophthalmology, Memorial Institute for Ophthalmic Research, Giza, Egypt

Corresponding Author: Mohammed Othman Abdel Khalek El Sayed, Lecturer, Department of Ophthalmology, Faculty of Medicine, Beni Suef University, Beni Suef, Egypt, Phone: +00201021660166, e-mail: nabi_mar@yahoo.com
}

\section{Source of support: Nil}

Conflict of interest: None

\section{INTRODUCTION}

Keratoconus (KC) is a progressive, noninflammatory bilateral but (usually asymmetrical) ecstatic corneal disease, characterized by paraxial stromal thinning and weakening that leads to corneal surface distortion. Visual disturbance occurs primarily from irregular astigmatism and myopia, and secondarily from corneal scarring. ${ }^{1}$

The reported prevalence of keratoconus varies widely depending upon the geographic location, diagnostic criteria used, and the cohort of patients selected. The prevalence in studies can range from 0.3 per 100,000 in Russia to 2,300 per 100,000 in Central India. A relatively high incidence has been documented in the Mediterranean and Middle Eastern areas. ${ }^{2}$

Histopathological changes in keratoconus starts with decrease in the keratocyte density, with reduction in the number of lamellae and degradation of fibroblasts in corneal stroma that leads to changes in the gross organization of the lamellae and an uneven distribution of the collagen fibrillar mass, especially around the apex of the cone. Structural abnormalities were occurred in the central part of Bowman's layer (BM) in the form of a sharp edge defects and interruption in the BM resulting from collagen bundle separation. ${ }^{3}$

Computer-assisted videokeratography (corneal topography) was one of the recent methods of diagnosis of keratoconus which depends on screening for keratoconic corneal shape changes. It depends on Rabinowitz diagnostic indices for diagnosis of keratoconus. The first indices were keratometric value (K value) which quantifies the central steepening of the cornea, a value more than $47.3 \mathrm{D}$ is suggestive of keratoconus. The second indices were (I-S value) which quantifies the inferior vs superior corneal dioptric asymmetry, a value of $1.4 \mathrm{D}$ or greater is suggestive of keratoconus. The last one and the most sensitive is (Keratoconus inferior-superior asymmetry\%) which is the keratometer inferior vs superior astigmatism where value greater than $100 \%$ is highly suggestive of frank keratoconus, and a range from 60 to $100 \%$ represents keratoconus suspects. Orbscan topographic system was the first system that scanned both the anterior and posterior surface of the cornea, so new parameters 
suggestive of posterior corneal elevation are postulated which add to the diagnosis of early keratoconus. Posterior corneal changes are considered to be suggestive of keratoconus, if one or more of these parameters are met, the ratio of radii of the anterior to posterior curvature: 1.21-1.27, the posterior best fit sphere more than $50 \mathrm{D}$, the difference between the thinnest and the thickest corneal pachymetry at $7 \mathrm{~mm}$ zone more than $90 \mathrm{~mm}$, the posterior corneal elevation more than $50 \mathrm{~mm}^{4}$

The Pentacam was originally introduced as an anterior segment analyzer that utilizes the Scheimpflug photography technique. During acquisition with Pentacam, which takes up to 2 seconds, a rotating Scheimpflug camera photographs cross section of the anterior segment which is illuminated by slit lights at different meridians. The Pentacam calculates a three-dimensional (3D) mathematical model of the anterior segment of the eye to provide the anterior and posterior corneal elevation maps, the corneal pachymetry from limbus to limbus, a 3D chamber analyzing (anterior chamber depth map, chamber angle, chamber volume). The most common display is a four map display that presents the refractive four- map display, where clockwise from the top left, the sagittal power, anterior elevation, posterior elevation (PE), and pachymetry maps are included. ${ }^{5}$

The Pentacam software compares the measured values with the means and standard deviations of a normal population, and helps provide color-coded "flags". For example, measured values which exceed the standard deviation by a factor of more than 2.5 are classified as abnormal and highlighted in yellow, and pathological values, i.e., values that exceed the standard deviation by a factor of more than three, are highlighted in red. Namely, these indices are the following:

- Index of surface variance (ISV)

- Index of vertical asymmetry (IVA)

- Keratoconus indices (KI)

- Central keratoconus indices (CKI)

- Index of height asymmetry (IHA)

- Index of height deviation (IHD)

- Minimum radius of curvature (Rmin).

Many lines of treatment are used to manage keratoconus according to its grade, starting by nonsurgical methods as spectacles, contact lens either soft or hard or by surgical interventional whether, by corneal collagen cross-linking, corneal ring segment inserts (INTACS) ends up to lamellar keratoplasty or full thickness penetrating keratoplasty. ${ }^{6}$

\section{AIM}

To estimate the incidence and indices of keratoconus in the patients seeking refractive procedures Beni Suef Governorate in the North of Upper Egypt. To describe and analyze the characteristics of the keratoconus population under study.

\section{MATERIALS AND METHODS}

This study was approved by the Medical Ethics Committee of Beni Suef University. A prospective study was made of all clinical records in the computerized database with the clinical diagnosis of keratoconus and/or suspected keratoconus between March 2013 and March 2014.

\section{Inclusion Criteria}

All patients were seeking a refractive procedure with minimum age 18 years.

\section{Exclusion Criteria}

- Patients less than 18 years old

- Patients with history of ophthalmic surgery

- Patients with history of autoimmune disease which leads to corneal thinning or severe dry eye

- History of chemical injury or delayed epithelial healing

- Pregnancy or lactation for female patients.

The diagnosis of keratoconus was based on the clinical examination of the anterior segment by the slit lamp to detect corneal keratoconus signs (Fleischer's ring, Vogts striae, and Munson's sign) and retinoscope assessment to detect scissoring or oil droplet sign and the diagnosis was confirmed by anterior segment imaging by corneal topographic images with Pentacam HR premium: WaveLight Allegro Oculyzer II (WaveLight AG, Germany). The Pentacam measurements were obtained and processed via the examination software (version 1.17r47) for each eye. Three consecutive measurements were obtained and processed to test for data acquisition repeatability.

Anterior segment, including anterior segment imaging measurement, was assessed:

- Flat corneal curvature K1 and the steep corneal curvature $\mathrm{K} 2$

- Keratometric reading

- Corneal thickness (thinnest location)

- Posterior surface elevation (central point and highest point in the central $5 \mathrm{~mm}$ )

- The minimum radius of curvature

- Keratometric power difference (KPD)

- Keratometric indices (7 indices)

\section{Index of Surface Variance}

It is an expression of the corneal surface irregularity. Index of surface variance greater than 37 is considered abnormal (marked with yellow) and greater than 41 is pathological (marked with red).

\section{Index of Vertical Asymmetry}

It measures in $\mathrm{mm}$ the mean difference between superior and inferior corneal curvature, so it is the value 
of curvature symmetry with respect to the horizontal meridian. Index of vertical asymmetry greater than 0.28 is considered abnormal and greater than 0.23 is pathological.

\section{Keratoconus Indices}

An index for expressing the ratio between mean radius values in the upper and lower segment. Keratoconus indices value greater than 1.07 is considered abnormal and/or pathological.

\section{Central Keratoconus Indices}

It is a ratio between mean radius values in a peripheral ring divided by central ring. Central keratoconus indices increase with the severity of central keratoconus. Central keratoconus indices value greater than 1.03 is considered abnormal and/or pathological.

\section{Index of Height Asymmetry}

It is the mean difference between height values superior minus height values inferior with the horizontal meridian as mirror axis (expressed in $\mu \mathrm{m}$ ). Index of height asymmetry is similar to the IVA but based on corneal elevation, and is thus more sensitive. Index of height asymmetry value greater than 19 is considered abnormal and greater than 21 is pathological.

\section{Index of Height Deviation}

This index provides the degree of decentration in the vertical direction and is calculated on a ring with radius $3 \mathrm{~mm}$. An IHD value more than 0.014 is considered abnormal and values greater than 0.016 is pathological.

\section{Minimum Radius of Curvature}

It measures in $\mathrm{mm}$ the smallest radius of sagittal corneal curvature (i.e., the maximum steepness of the cone). Values of Rmin less than $6.71 \mathrm{~mm}$ are considered abnormal and/or pathological.

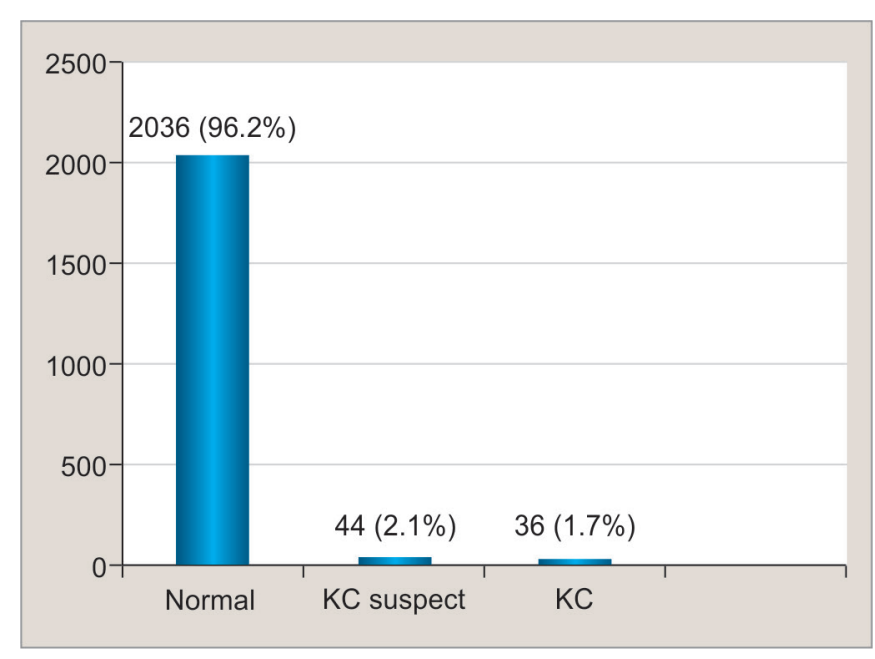

Graph 1: Normal vs keratoconus suspect vs keratoconus
All data will be collected, tabulated, and analyzed using IBM Statistical Package for the Social Sciences statistics version 20 (SPSS Inc., Chicago, IL, USA) software.

\section{RESULTS}

After applying of the exclusion criteria, data from 2,116 cases with the average $28.8 \pm 6.5$ years old were analyzed. These cases were studied over the span of 1 year starting from March 2013 to March 2014. In this study sample, 1,249 cases were female (59\%) and 867 cases (41\%) were male. Based on topographic images of the Pentacam, 36 eyes were diagnosed as keratoconus. The incidence of keratoconus was $1.7 \%$ (95\% confidence interval, $0.55-1.45)$. A total of 11 patients (62\%) were diagnosed as bilateral keratoconus, 7 patients (68\%) were female and 4 patients $(32 \%)$ were male, and 14 patients $(38 \%)$ were diagnosed as unilateral keratoconus, 8 patients $(57 \%)$ were female and6 patients $(43 \%)$ were male (Table 1$)$.

The diagnosis of keratoconus was topographic confirmed in 36 eyes $(1.7 \%)$. The keratoconus suspects were 44 cases $(2.1 \%)$ and 2,036 cases were normal (Graphs 1 and 2)

In keratoconic patients, the mean age was 28.1 years, the mean $\mathrm{K} 1$ and $\mathrm{K} 2$ were $45.18 \pm 3.44 \mathrm{D}$ and $49.49 \pm 4.28$ $D$ respectively. The mean minimal pachymetry value was $463 \pm 6.51 \mu \mathrm{m}$, the mean ISV was $61 \pm 32$, the mean IVA was $0.63 \pm 0.38$, and the mean KI was $1.08 \pm 0.14$ (Table 2).

According to the Pentacam software keratoconus classification:

The incidence of different grades of keratoconus (Table 2):

\begin{tabular}{llll}
\hline & Number & Percentage & Prevalence \\
\hline KC1 & 8 & $24 \%$ & 0.003 \\
KC1-2 & 6 & $16 \%$ & 0.002 \\
KC2 & 9 & $26 \%$ & 0.004 \\
KC2-3 & 4 & $11 \%$ & 0.001 \\
KC3 & 5 & $12 \%$ & 0.002 \\
KC3-4 & 4 & $11 \%$ & 0.001 \\
\hline Total & 36 & $100 \%$ & 0.013 \\
\hline
\end{tabular}

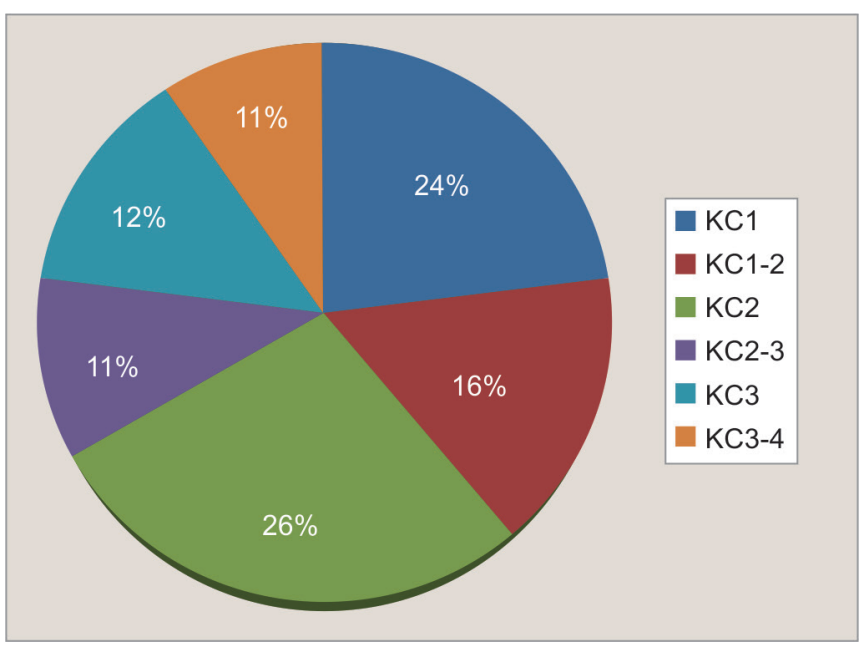

Graph 2: The percentage of different grades of keratoconus 
Table 1: Demographic distribution of keratoconus patients

\begin{tabular}{lll}
\hline & Bilateral KC & Unilateral KC \\
\hline Female & $7(68 \%)$ & $8(57 \%)$ \\
Male & $4(32 \%)$ & $6(43 \%)$ \\
\hline
\end{tabular}

Table 2: Values of Pentacam (mean, median, minimum, and maximum) of the keratoconic patients

\begin{tabular}{llllll}
\hline \multicolumn{5}{c}{ Standard } & \\
& Mean & deviation & Median & Minimum & Maximum \\
\hline Age & 28.16 & 9.85 & 26 & 18 & 50 \\
K1 & 45.18 & 3.44 & 45.00 & 36.4 & 59.1 \\
K2 & 49.49 & 4.28 & 49 & 39.6 & 67.9 \\
Thinnest & 463.37 & 66.51 & 476.00 & 124.00 & 573.00 \\
location & & & & & \\
ISV & 60 & 32 & 56 & 1 & 170 \\
IVA & 0.63 & 0.38 & 0.51 & 0.09 & 1.62 \\
KI & 1.08 & 0.14 & 1.07 & 0.78 & 1.44 \\
CKI & 1.03 & 0.05 & 1.02 & 0.87 & 1.23 \\
IHA & 25 & 22 & 18.8 & 0.1 & 116 \\
IHD & 0.059 & 0.046 & 0.042 & 0.002 & 0.24 \\
Rmin & 6.28 & 0.86 & 6.49 & 0.31 & 7.71 \\
KPD & 1.61 & 0.74 & 1.4 & 0.6 & 4.8 \\
Prog Index & 2.14 & 3.4 & 1.6 & 0 & 35.5 \\
axis & & & & & \\
\hline
\end{tabular}

Descriptive statistics for the keratoconus grading subgroups for age and the seven anterior surface topometric indices (Tables 3 to 5).

The PE measurement in the 3-mm zone had the strongest power to distinguish keratoconus from normal (Graph 3). Optimal cutoff point for PE in the 3-mm zone was $18.5 \mu \mathrm{m}$ for keratoconus (sensitivity, $92 \%$; specificity, 95\%).

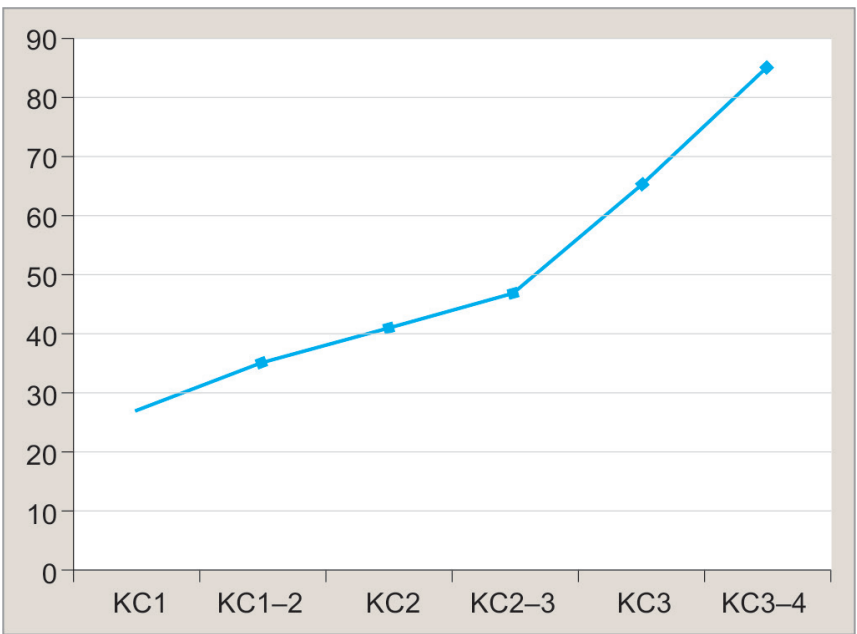

Graph 3: Mean posterior elevation for each keratoconus grade

Table 3: Descriptive statistics keratoconus grading

\begin{tabular}{|c|c|c|c|c|c|c|c|}
\hline & Stage I & Stage I-II & Stage II & Stage II-III & Stage III & Stage III-IV & Stage IV \\
\hline \multicolumn{8}{|l|}{ Age } \\
\hline Mean & 31.0 & 32.5 & 31.3 & 31.3 & 30.9 & 30.6 & 31.0 \\
\hline $\mathrm{SD}( \pm)$ & 8.4 & 7.6 & 7.8 & 6.0 & 6.7 & 7.3 & 8.4 \\
\hline \multicolumn{8}{|c|}{ Index of surface variance } \\
\hline Mean & 40.6 & 50.91 & 72.75 & 93.77 & 114.07 & 157.8 & 218.8 \\
\hline $\mathrm{SD}( \pm)$ & 2.88 & 3.56 & 9.32 & 5.25 & 9.51 & 17.9 & 29 \\
\hline SEM & 0.91 & 1.1 & 1.6 & 1.1 & 1.3 & 2.7 & 14 \\
\hline \multicolumn{8}{|c|}{ Index of vertical asymmetry } \\
\hline Mean & 0.422 & 0.5618 & 0.855 & 1.068 & 1.219 & 1.612 & 2.002 \\
\hline $\mathrm{SD}( \pm)$ & 0.0735 & 0.0994 & 0.134 & 0.194 & 0.258 & 0.361 & 0.497 \\
\hline SEM & 0.023 & 0.03 & 0.024 & 0.041 & 0.035 & 0.054 & 0.25 \\
\hline \multicolumn{8}{|c|}{ Keratoconus indices } \\
\hline Mean & 1.10 & 1.12 & 1.1838 & 1.2664 & 1.3291 & 1.47 & 1.7475 \\
\hline $\mathrm{SD}( \pm)$ & 0.0163 & 0.0335 & 0.0522 & 0.027 & 0.0547 & 0.121 & 0.0929 \\
\hline SEM & 0.0052 & 0.01 & 0.0092 & 0.0058 & 0.0075 & 0.018 & 0.046 \\
\hline \multicolumn{8}{|c|}{ Central keratoconus indices } \\
\hline Mean & 1.014 & 1.0145 & 1.0291 & 1.05 & 1.0711 & 1.1269 & 1.208 \\
\hline $\mathrm{SD}( \pm)$ & 0.0135 & 0.023 & 0.0352 & 0.0385 & 0.0394 & 0.0702 & 0.101 \\
\hline SEM & 0.0043 & 0.0069 & 0.0062 & 0.0082 & 0.0054 & 0.01 & 0.051 \\
\hline \multicolumn{8}{|c|}{ Index of height asymmetry } \\
\hline Mean & 18.05 & 20.55 & 26.5 & 33.3 & 37.7 & 39.3 & 61.7 \\
\hline $\mathrm{SD}( \pm)$ & 9.66 & 7.68 & 15.6 & 17.3 & 19.3 & 28.8 & 14.4 \\
\hline SEM & 3.1 & 2.3 & 2.7 & 3.7 & 2.6 & 4.3 & 7.2 \\
\hline \multicolumn{8}{|c|}{ Index of height decentration } \\
\hline Mean & 0.031 & 0.04355 & 0.0677 & 0.0878 & 0.1063 & 0.1512 & 0.24 \\
\hline $\operatorname{SD}( \pm)$ & 0.00723 & 0.00972 & 0.0183 & 0.0169 & 0.0266 & 0.0373 & 0.0185 \\
\hline SEM & 0.0023 & 0.0029 & 0.0032 & 0.0036 & 0.0036 & 0.0056 & 0.0093 \\
\hline \multicolumn{8}{|c|}{ Minimum radius of curvature $(\mathrm{mm})$} \\
\hline Mean & 6.922 & 6.755 & 6.52 & 6.104 & 5.775 & 5.146 & 4.165 \\
\hline $\operatorname{SD}( \pm)$ & 0.311 & 0.275 & 0.463 & 0.483 & 0.381 & 0.675 & 0.513 \\
\hline SEM & 0.098 & 0.083 & 0.082 & 0.1 & 0.052 & 0.1 & 0.26 \\
\hline
\end{tabular}


Incidence and Indices of Keratoconus in Patients presenting for LASIK in Egypt

Table 4: The values of age [mean, median, and standard deviation (SD)] according to different grades of keratoconus

\begin{tabular}{lllll}
\hline & \multicolumn{3}{c}{ Age } \\
\cline { 2 - 5 } & Number & Mean & Median & SD \\
\hline KC1 & 8 & 26.19 & 24.5 & 9.26 \\
KC1-2 & 6 & 27.73 & 24.00 & 12.23 \\
KC2 & 9 & 28.11 & 26.00 & 8.26 \\
KC2-3 & 4 & 21.75 & 18.5 & 8.00 \\
KC3 & 5 & 25.78 & 25.00 & 7.71 \\
KC3-4 & 4 & 22.71 & 22.00 & 8.28 \\
\hline
\end{tabular}

\section{DISCUSSION}

Keratoconus is a corneal degeneration characterized by stromal thinning and conical ectasia. The incidence of keratoconus among refractive surgery candidate has been reported to vary from 0.9 to $8.1 \%$. The Pentacam which is based on the Scheimpflug principle, measures 138,000 elevation points. This technique can be used as a screening test for detecting keratoconus since it works independent of axis, orientation, and position of the eye. It has been shown that elevation-based topography systems are more accurate than placid-based devices in differentiating normal eyes from early keratoconus. ${ }^{\text {? }}$

This prospective surveillance study was conducted in Beni Suef Governorate in the north of Upper Egypt. After applying of the exclusion criteria, data from 2,116 cases with the average $28.8 \pm 6.5$ years were analyzed. These cases were studied over the span of 1 year starting from March 2013 to March 2014. In this study sample, 1,249 cases were female $(59 \%)$ and $867(41 \%)$ were male. Based on topographic images of Pentacam, 36 eyes were diagnosed as keratoconus. The incidence of keratoconus was $1.7 \%$ (95\% confidence interval, $0.55-1.45)$. A total of 11 patients $(62 \%)$ were diagnosed as bilateral keratoconus, $7(68 \%)$ females and $4(32 \%)$ males. The keratoconus suspects were 44 cases $(2.1 \%)$ and 2,036 cases were normal. The mean K1 and K2 were $45.18 \pm 3.44 \mathrm{D}$ and $49.49 \pm 4.28$ D respectively. The mean minimal pachymetry value was $463 \pm 6.51 \mu \mathrm{m}$, the mean ISV was $61 \pm 32$, the mean IVA was $0.63 \pm 0.38$, and the mean KI was $1.08 \pm 0.14$.

Ziaei et $\mathrm{al}^{8}$ did a prospective surveillance study in Yazd province in the central Iran to determine the incidence of keratoconus. This study was conducted on 564 cases from July 2008 to June 2009. Based on Pentacam topographic images, keratoconus was confirmed in 22 cases $(2.5 \%)$. These results are comparable to ours.

Hashemi et $\mathrm{al}^{9}$ did a study to determine the incidence of keratoconus in an Iranian population selected from Tehran city. Orbscan topographic maps were acquired from 4,592 cases with the mean age of $50.83 \pm 0.12$ years. Thirty-five patients were diagnosed with keratoconus and the prevalence of keratoconus was $0.76 \%$ (95\% confidence interval, 0.35-1.09) with the mean age was
Table 5: Mean maximum, and minimum posterior elevation (PE) for each keratoconus grade

\begin{tabular}{llll}
\hline & Mean PE & Minimum & Maximum \\
\hline KC1 & 27 & 19 & 33 \\
KC1-2 & 35 & 35 & 39 \\
KC2 & 41 & 40 & 45 \\
KC2-3 & 47 & 47 & 61 \\
KC3 & 65 & 63 & 75 \\
KC3-4 & 85 & 75 & 102 \\
\hline
\end{tabular}

47.6 years. Keratoconus suspects were 47 cases (1.02\%). The mean age of keratoconic patients was 47.6 years. The mean maximum keratometry and the central corneal thickness in patients with keratoconus were $48.67 \pm 3.6$ diopter and $470 \pm 29 \mu \mathrm{m}$ respectively. The higher rates in prevalence of keratoconus rate in our study compared to this study can be explained by topographic maps acquisition by Pentacam rather than Orbscan which used in this study.

Mohd-Ali et $\mathrm{al}{ }^{10}$ published a survey study to evaluate the incidence of keratoconus in Malaysia. Records from 13,000 patients were reviewed. A total of 159 patients had keratoconus and the prevalence was $1.2 \%$. The mean age of keratoconus was $20.9 \pm 5.6$ years with $71.1 \%$ males and $28.9 \%$ females.

Serdarogullari et $\mathrm{al}^{11}$ did a study to determine the incidence of keratoconus in Turkish population and compared the Pentacam parameters among these subjects. Around 128 eyes of 64 subjects with astigmatism greater than $2 \mathrm{D}$ were included in this study. From the entire study sample, 8 eyes (6.3\%) were diagnosed with keratoconus and 10 eyes $(7.8 \%)$ as subclinical keratoconus. The mean age was $32.1 \pm 7.3$ years in patients with keratoconus. The mean minimal pachymetry was $463 \pm 52 \mu \mathrm{m}$ in keratoconus eyes. The mean PE was $39.88 \pm 15.76 \mu \mathrm{m}$. The high prevalence of keratoconus in this study may be due to small number of patients in this study sample or difference in the study sample species.

Assiri et $\mathrm{al}^{12}$ did a study to estimate the incidence of keratoconus in Asir province in KSA, the incidence was 20 cases per 100,000 population. Also, the disease severity is high, as indicated by an early mean age [17.7 (3.6) years] with advanced stage keratoconus.

\section{CONCLUSION}

This study provides the first population-based estimate of the incidence of keratoconus in Beni Suef Governorate in the Upper Egypt. According to this study, the incidence rate of keratoconus is comparable to the rate among the Caucasian people in Middle Asia, e.g., Islamic Republic of Iran whereas incidence rate is much higher among Malaysia and KSA citizens. 


\section{REFERENCES}

1. Rabinowitz YS, Klyce SD, Krachmer JH. Keratoconus, videokeratography, and refractive surgery. Refract Corneal Surg 1992 Sep-Oct;8(5):403-407.

2. Jonas JB, Nangia V, Matin A, Kulkarni M, Bhojwani K. Prevalence and associations of keratoconus in rural Maharashtra in central India: the central India eye and medical study. Am J Ophthalmol 2009 Nov;148(5):760-765.

3. Meek KM, Tuft SJ, Huang Y, Gill PS, Hayes S, Newton RH, Bron AJ. Changes in collagen orientation and distribution in keratoconus corneas. Invest Ophthalmol Vis Sci 2005 Jun;46(6):1948-1956.

4. Kawanaa K, Tokunaga T, Miyata K, Okamoto F, Kiuchi T, Oshika T. Comparison of corneal thickness measurements using Orbscan II, non-contact specular microscopy, and ultrasonic pachymetry in eyes after laser in situ keratomileusis. Br J Ophthalmol 2004 Apr;88(4);466-468.

5. Verges C, Cazal J. 2009. Applications of Pentacam in anterior segment analysis; highlights of ophthalmology. [Internet] [cited 2017 February]. Available from: www.oculus.de/pl/ downloads/dyn/oculus/presse/172/verges_english.pdf.
6. Vazirani J, Basu S. Keratoconus: current perspectives. Clin Ophthalmol 2013;1:2019-2030.

7. Jafri B, Li X, Yang H, Rabinowitz YS. Higher order wavefront aberrations and topography in early and suspected keratoconus. J Refract Surg 2007 Oct;23(8):774-781.

8. Ziaei H, Jafarinasab MR, Javadi MA, Karimian F, Poorsalman H, Mahdavi M, Shoja MR, Katibeh M. Epidemiology of keratoconus in an Iranian population. Cornea 2012 Sep;31(9):1044-1047.

9. Hashemi H, Khabazkhoob M Fotouhi A. Topographic Keratoconus is not Rare in an Iranian population: the Tehran Eye Study. Ophthalmic Epidemiol 2013 Dec;20(6):385-391.

10. Mohd-Ali B, Abdu M, Yaw CY, Mohidin N. Clinical characteristics of keratoconus patients in Malaysia: a review from a cornea specialist centre. J Optom 2012 Jan;5(1):38-42.

11. Serdarogullari H, Tetikoglu M, Karahan H, Altin F, Elcioglu M. Prevalence of Keratoconus and Subclinical Keratoconus in Subjects with Astigmatism Using Pentacam Derived Parameters. J Ophthalmic Vis Res. 2013 Jul;8(3):213-219.

12. Assiri AA, Yousuf BI, Quantock AJ, Murphy PJ. Incidence and severity of keratoconus in Asir province, Saudi Arabia. Br J Ophthalmol 2005 Nov;89(11):1403-1406. 